\title{
Adaptive Direction-of-Arrival Estimation in Array Signal Processing
}

\author{
Jingmin Xin ${ }^{\dagger}$ and Akira Sano ${ }^{\S}$ \\ ${ }^{\dagger}$ Fujitsu Laboratories Ltd., 5-5 Hikari-no-oka, Yokosuka 239-0847, Japan \\ $\S$ Department of System Design Engineering, Keio University \\ 3-14-1 Hiyoshi, Kohoku-ku, Yokohama 223-8522, Japan
}

\begin{abstract}
A new computationally efficient subspacebased method without eigendecomposition (SUMWE) is investigated for the direction estimation of coherent narrowband signals impinging on a uniform linear array by exploiting the array geometry and its shift invariance property. In the proposed method, the computationally cumbersome eigendecomposition and the evaluation of all correlations of the array data are not required, and the effect of additive noise is eliminated. And the SUMWE can be extended to the spatially correlated noise by choosing appropriate subarrays. Further an adaptive implementation of the SUMWE is presented for tracking the directions of slowly moving signals. The performance of the presented algorithm is verified through numerical examples.
\end{abstract}

\section{Introduction}

The directions-of-arrival (DOAs) estimation of signals impinging on an array of sensors is a fundamental problem in many fields (e.g. [1], [2]). For the direction estimation of narrowband signals, the maximum likelihood (ML) and subspace-based methods have been studied extensively (see [1], [2] and references therein). The advantage of most subspace-based methods (e.g. multiple signal classification (MUSIC) [3]) over the ML methods is their relatively computational simplicity, where the DOAs are estimated through the search of an one-dimensional spectrum or the calculation of the roots of a certain polynomial based on either the eigenvalue decomposition (EVD) of an array covariance matrix or the singular value decomposition (SVD) of a matrix of array data. Unfortunately, the eigendecomposition process is still computationally intensive and time-consuming, which means that subspace-based methods are unsuitable for some practical situations when the number of sensors is large and/or the directions of impinging signals should be tracked in an on-line manner [4], because they require repeated EVD/SVD to update the signal/noise subspace with the acquisition of new data and the deletion of the old data.

For alleviating the difficulty of subspace-based methods, some computationally simple subspace-based direction estimation methods without eigendecomposition have been developed [5]-[8]. In linear operation based methods such as the bearing estimation without eigendecomposition (BEWE) [5], orthonormal propagator method (OPM) [6], and subspace methods without eigendecomposition (SWEDE) [7], the signal or noise subspace is easily obtained from the array data relying on a partition of array response matrix, and then the directions are estimated in a manner similar to that of the MUSIC [3]. Unfortunately, their accuracy is generally poorer than that of the conventional subspace-based methods (e.g. MUSIC) from the statistical viewpoint [9], [6], [7]. The WSF without eigendecomposition (WSF-E) [8] achieves the asymptotic efficiency when either the number of snapshots or the signal-to-noise ratio (SNR) is large, but they are computationally much more complicated than linear operation based algorithms [5]-[7]. Furthermore, most of these computationally simple subspace-based methods suffer serious degradation when the incident signals are coherent (i.e. fully correlated) in some practical scenarios due to multipath propagation (e.g. [18]), where the rank of the source signal covariance matrix becomes less than the number of incident signals. Although the WSF-E [8] and a variant of BEWE [5] can resolve the coherent signals, their performance degrades severely at low SNR and with a small number of snapshots.

Therefore we propose a new computationally efficient subspace-based method without eigendecomposition (SUMWE) for the direction estimation of coherent narrowband signals impinging on a uniform linear array (ULA) by exploiting the array geometry and its shift invariance property. The SUMWE does not require the computationally cumbersome eigendecomposition and the evaluation of all correlations of the array data, and the effect of additive noise is eliminated. Moreover, the SUMWE is also suitable for the case of partly coherent or incoherent signals, and it can be extended to the spatially correlated noise by choosing appropriate subarrays (i.e. cross-correlations of array data). Furthermore, an adaptive implementation of the SUMWE is presented for tracking the directions of slowly moving (relative to the sampling rate) signals. The performance of the presented algorithm is verified and compared with the conventional methods, and it is shown that the proposed algorithm is computationally simple and has a good tracking ability.

\section{Data Model and Basic Assumptions}

Consider a ULA of $M$ identical and omnidirectional sensors with spacing $d$, and suppose that $p$ narrowband signals $\left\{s_{k}(n)\right\}$ with the centre frequency $f_{0}$ are in the field far from the array and impinge on the array from distinct directions $\left\{\theta_{k}(n)\right\}$. Under the narrowband assumption, the received noisy signal $y_{i}(n)$ at the $i$ th sensor can be expressed as [1], [2], [11]-[13]

$$
\begin{aligned}
& y_{i}(n)=x_{i}(n)+w_{i}(n) \\
& x_{i}(n)=\sum_{k=1}^{p} s_{k}(n) e^{j \nu_{0}(i-1) \tau\left(\theta_{k}(n)\right)}
\end{aligned}
$$

where $x_{i}(n)$ is the noiseless received signal, $w_{i}(n)$ is the additive noise, $\omega_{0} \triangleq 2 \pi f_{0}, \tau\left(\theta_{k}(n)\right) \triangleq(d / c) \sin \theta_{k}(n), \quad c \quad$ is the propagation speed, and $\left\{\theta_{k}(n)\right\}$ are measured relative 
to the normal of array. The received signals can be reexpressed more compactly as

$$
y(n)=A(\theta) s(n)+w(n)
$$

where $y(n), s(n)$, and $w(n)$ are the vectors of the received signals, the incident signals, and the additive noise, $A(\theta)$ is the array response matrix given by $A(\theta) \triangleq\left[a\left(\theta_{1}(n)\right), a\left(\theta_{2}(n)\right), \cdots, a\left(\theta_{p}(n)\right)\right] \quad$ with $\quad a\left(\theta_{k}(n)\right) \triangleq[1$, $\left.e^{j \omega_{0} \tau\left(\theta_{k}(n)\right)}, \cdots, e^{j \omega_{n}(M-1) \tau\left(\theta_{k}(n)\right)}\right]^{T}$, and $(\cdot)^{T}$ denotes the transpose.

In this paper, we make the following basic assumptions on the data model.

A1: The array is calibrated and the array response matrix $A(\theta)$ is unambiguous, i.e. the array response vectors $\left\{a\left(\theta_{1}(n)\right), a\left(\theta_{2}(n)\right), \cdots, a\left(\theta_{p}(n)\right)\right\} \quad$ are linearly independent for any set of distinct $\left\{\theta_{1}(n), \theta_{2}(n), \cdots, \theta_{p}(n)\right\}$. Equivalently $A(\theta)$ has full rank.

$A 2$ : The signals $\left\{s_{k}(n)\right\}$ are all coherent so that they are all some complex multiples of a common signal $s_{1}(n)$, then under the flat-fading multipath propagation, they can be expressed as [10]-[14], [18]

$$
s_{k}(n)=\beta_{k} s_{1}(n), \quad \text { for } k=1,2, \cdots, p
$$

where $\beta_{k}$ is the complex attenuation coefficient with $\beta_{k} \neq 0$ and $\beta_{1}=1$.

A3: For the simplicity of theoretical performance analysis, the incident signal $s_{1}(n)$ is a temporally complex white Gaussian random process with zero-mean and the variance given by

$$
E\left\{s_{1}(n) s_{1}^{*}(t)\right\}=r_{s} \delta_{n, t}, \quad E\left\{s_{1}(n) s_{1}(t)\right\}=0
$$

where $E\{\cdot\},(\cdot)^{*}$, and $\delta_{n, t}$ denote the expectation, the complex conjugate, and Kronecker delta.

A4: The additive noise $\left\{w_{i}(n)\right\}$ is a temporally and spatially complex white Guassian random process with zero-mean and the following covariance matrix

$$
E\left\{\boldsymbol{w}(n) \boldsymbol{w}^{H}(t)\right\}=\sigma^{2} I_{M} \delta_{n, t}, \quad E\left\{\boldsymbol{w}(n) \boldsymbol{w}^{T}(t)\right\}=O_{M \times M}
$$

where $I_{m}, O_{m \times q}$, and $(\cdot)^{H}$ indicate the $m \times m$ identity matrix, the $m \times q$ null matrix, and Hermitian transpose. And the noise is uncorrelated with the incident signals.

A5: The number of incident signals $p$ is known or estimated by some proposed techniques (e.g. [11] and references therein)., and it satisfies the inequality that $p<M / 2$ for an array of $M$ sensors.

\section{Subspace-Based Direction Estimation Method without Eigendecomposition - SUMWE}

\subsection{Decorrelation with Subarray Averaging}

Here we consider the estimation of constant directions of coherent signals, where $\theta_{k}(n)=\theta_{k}$. First, the noiseless signal $x_{i}(n)$ in (2) can be reexpressed by

$$
x_{i}(n)=b_{i}^{T}(\theta) s(n)=s^{T}(n) b_{i}(\theta)
$$

where $b_{i}(\theta) \triangleq\left[e^{j \omega_{0}(i-1) r\left(\theta_{1}\right)}, e^{j \omega_{0}(i-1) \tau\left(\theta_{2}\right)}, \cdots, e^{j \omega_{0}(i-1) \tau\left(\theta_{p}\right)}\right]^{T}$, and the correlation $r_{i k}$ of signals $y_{i}(n)$ and $y_{k}(n)$ is defined as $r_{i k} \triangleq E\left\{y_{i}(n) y_{k}^{*}(n)\right\}$, where $r_{i k}=r_{k i}^{*}$. Then the full array can be divided into $L$ overlapping subarrays with $p$ sensors in the forward and backward directions [10], [14], where $L=M-p+1$, and the $l$ th forward or backward subarray comprises $\{l, l+1, \cdots, l+p-1\}$ or $\{M-l+1, M-l, \cdots, L-l$ $+1\}$ sensors, where $l=1,2, \cdots, L$, and we can express the signal vector $y_{j}(n)$ of the signals in the $l$ th forward subarray and that $y_{b l}(n)$ of the conjugate signals in the $l$ th backward subarray as [10]-[14]

$$
\begin{aligned}
& y_{f l}(n)=A_{1}(\theta) D^{l-1} s(n)+w_{f l}(n) \\
& y_{b l}(n)=A_{1}(\theta) D^{-(M-l)} s^{*}(n)+w_{b l}(n)
\end{aligned}
$$

where $\quad y_{f l}(n) \triangleq\left[y_{l}(n), y_{l+1}(n), \cdots, y_{l+p-1}(n)\right]^{T} \quad, \quad y_{b l}(n) \triangleq$ $\left[y_{M-l+1}(n), y_{M-l}(n), \cdots, y_{L-l+1}(n)\right]^{H}, \quad w_{l}(n) \triangleq\left[w_{l}(n), w_{l+1}(n), \cdots\right.$, $\left.w_{l+p-1}(n)\right]^{T}, \quad w_{b l}(n) \triangleq\left[w_{M-l+1}(n), w_{M-l}(n), \cdots, w_{L-l+1}(n)\right]^{H}, \quad D \triangleq$ $\operatorname{diag}\left(e^{j \omega_{0} r\left(\theta_{1}\right)}, e^{j \omega_{0} \tau\left(\theta_{2}\right)}, \cdots, e^{j \omega_{0} \tau\left(\theta_{p}\right)}\right)$, and $A_{1}(\theta)$ is the submatrix of $A(\theta)$ in (3) consisting of the first $p$ rows with the column $a_{1}\left(\theta_{k}\right) \triangleq\left[1, e^{j \omega_{0} r\left(\theta_{k}\right)}, \cdots, e^{j \omega_{0}(p-1) \tau\left(\theta_{k}\right)}\right]^{T}$.

Under the basic assumptions, from (4)-(8), we can obtain the correlation $\varphi_{f}$ between the signal vector $y_{f}(n)$ of the $l$ th forward subarray and the signal $y_{M}^{*}(n)$

$$
\begin{aligned}
\varphi_{f} & \triangleq E\left\{y_{f}(n) y_{M}^{*}(n)\right\}=\left[r_{M}, r_{i+1, M}, \cdots, r_{l+p-1, M}\right]^{T} \\
& =E\left\{A_{1}(\theta) \boldsymbol{D}^{\prime-1} \beta s_{1}(n) s_{1}^{*}(n) \boldsymbol{\beta}^{H} b_{M}^{*}(\theta)\right\}+E\left\{\boldsymbol{w}_{f}(n) w_{M}^{*}(n)\right\} \\
& =\rho_{M} r_{s} A_{1}(\theta) \boldsymbol{D}^{\prime-1} \boldsymbol{\beta}, \quad \text { for } l=1,2, \cdots, L-1
\end{aligned}
$$

where $\rho_{i} \triangleq \beta^{H} b_{i}^{*}(\theta)$, and $\beta \triangleq\left[\beta_{1}, \beta_{2}, \cdots, \beta_{p}\right]^{T}$. From (10) and by performing some manipulations, we can form a correlation matrix $\Phi_{f}$ as

$$
\Phi_{f} \triangleq\left[\varphi_{f 1}, \varphi_{f 2}, \cdots, \varphi_{f L-1}\right]^{r}=\rho_{M} r_{s} \bar{A}(\theta) B A_{1}^{T}(\theta)
$$

where $B \triangleq \operatorname{diag}\left(\beta_{1}, \beta_{2}, \cdots, \beta_{p}\right)$, and $\bar{A}(\theta)$ is the submatrix of $A(\theta)$ consisting of the first $L-1$ rows with the column $\bar{a}\left(\theta_{k}\right) \triangleq\left[1, e^{j \omega_{n} r\left(\theta_{k}\right)}, \cdots, e^{j \omega_{0}(L-2) \tau\left(\theta_{k}\right)}\right]^{r}$. Straightforwardly we can see that the Hankel matrix $\Phi_{f}$ is composed of the cross-correlations $\left\{r_{1 M}, r_{2 M}, \cdots, r_{M-1, M}\right\}$. Similarly we can get the correlation $\bar{\varphi}_{f l}$ between the signal vector $y_{n}(n)$ and the signal $y_{1}^{*}(n)$

$$
\begin{aligned}
\bar{\varphi}_{f} & \triangleq E\left\{\boldsymbol{y}_{f}(n) y_{1}^{\prime \prime}(n)\right\}=\left[r_{11}, r_{l+1,1}, \cdots, r_{l+p-1,1}\right]^{T} \\
& =\rho_{1} r_{s} A_{4}(\theta) D^{\prime-1} \beta, \quad \text { for } l=2,3, \cdots, L .
\end{aligned}
$$

And we can easily obtain a correlation matrix $\bar{\Phi}_{f}$ formed from the correlations $\left\{\bar{\varphi}_{f}\right\}$ in (12)

$$
\bar{\Phi}_{f} \triangleq\left[\bar{\varphi}_{f 2}, \bar{\varphi}_{f 3}, \cdots, \bar{\varphi}_{f L}\right]^{T}=\rho_{1} r_{s} \bar{A}(\theta) B D A_{l}^{T}(\theta) .
$$

Obviously the Hankel matrix $\bar{\Phi}_{f}$ consists of the cross-correlations $\left\{r_{21}, r_{31}, \cdots, r_{M 1}\right\}$.

By evaluating the correlation $\varphi_{b l} \triangleq E\left\{y_{1}(n) y_{b l}(n)\right\}$ between $y_{1}(n)$ and $\left\{y_{b l}(n)\right\}$ and the correlation $\bar{\varphi}_{b l} \triangleq$ $E\left\{y_{M}(n) y_{b l}(n)\right\}$ between $y_{M}(n)$ and $\left\{y_{b l}(n)\right\}$, we can obtain the correlation matrices $\boldsymbol{\Phi}_{b}$ and $\overline{\boldsymbol{\Phi}}_{b}$ for the backward subarrays

$$
\begin{aligned}
& \Phi_{b} \triangleq\left[\varphi_{b 1}, \varphi_{b 2}, \cdots, \varphi_{b L-1}\right]^{T}=\rho_{1}^{*} r_{s} \bar{A}(\theta) B^{*} D^{-(M-1)} A_{1}^{T}(\theta) \\
& \bar{\Phi}_{b} \triangleq\left[\bar{\varphi}_{b 2}, \bar{\varphi}_{b 3}, \cdots, \bar{\varphi}_{b L}\right]^{T}=\rho_{M}^{*} r_{s} \bar{A}(\theta) B^{*} D^{-(M-2)} A_{1}^{T}(\theta) .
\end{aligned}
$$

It is apparent that the Hankel matrix $\boldsymbol{\Phi}_{b}$ consists of the cross-correlations $\left\{r_{M}, r_{1, M-1}, \cdots, r_{2}\right\}$, while the Hankel matrix $\bar{\Phi}_{b}$ is formed by the cross-correlations $\left\{r_{M, M-1}\right.$, $\left.r_{M, M-2}, \cdots, r_{M 1}\right\}$. And we can easily find that $\bar{\Phi}_{b}=J_{L-1} \bar{\Phi}_{f}^{m} J_{p}$ and $\bar{\Phi}_{b}=J_{L-1} \Phi_{f}^{*} J_{p}$, where $J_{m}$ is an $m \times m$ counteridentity matrix.

From (11) and (13)-(15), we can easily see that the correlation matrices $\Phi_{f}, \bar{\Phi}_{f}, \Phi_{b}$, and $\bar{\Phi}_{b}$ are not affected by the additive noise $\left\{w_{i}(n)\right\}$. Because $M>2 p$, $\beta_{k} \neq 0$, and $A(\theta)$ is a Vandermonde matrix with full rank, we can find that the ranks of these $(L-1) \times p$ matrices equal $p[11]-[13]$, i.e. the dimension of their signal subspace equals the number of coherent signals. Hence the directions $\left\{\theta_{k}\right\}$ of coherent signals can be estimated from the subspaces of these matrices $\Phi_{f}, \bar{\Phi}_{f}, \Phi_{b}$, and $\bar{\Phi}_{b}$.

Remark A: When some of the incident signals are 
coherent and the others are uncorrelated with these signals and with each other, by assuming that the first $q$ $(1 \leq q \leq p)$ signals are coherent as defined in (4) and performing some algebraic manipulations, we can obtain

$$
\begin{aligned}
& \Phi_{f}=\bar{\rho}_{M} r_{s_{1}} \bar{A}(\theta) \bar{B} A_{1}^{T}(\theta)+\bar{A}(\theta) D^{-(M-1)} \tilde{R}_{s} A_{1}^{T}(\theta) \\
& \bar{\Phi}_{f}=\bar{\rho}_{1} r_{s_{1}} \bar{A}(\theta) \bar{B} D A_{1}^{T}(\theta)+\bar{A}(\theta) D \tilde{R}_{s} A_{1}^{T}(\theta) \\
& \Phi_{b}=\bar{\rho}_{1}^{*} r_{s 1} \bar{A}(\theta) \bar{B}^{*} D^{-(M-1)} A_{1}^{T}(\theta)+\bar{A}(\theta) D^{-(M-1)} \tilde{R}_{s} A_{1}^{T}(\theta) \\
& \bar{\Phi}_{b}=\bar{\rho}_{M}^{*} r_{s l} \bar{A}(\theta) \bar{B}^{*} D^{-(M-2)} A_{1}^{T}(\theta)+\bar{A}(\theta) D \tilde{R}_{s} A_{1}^{T}(\theta)
\end{aligned}
$$

where $r_{s k_{k}} \triangleq E\left\{s_{k}(n) s_{k}^{*}(n)\right\}, \quad \bar{\rho}_{i} \triangleq \overline{\boldsymbol{\beta}}^{H} b_{i}^{*}(\theta), \overline{\boldsymbol{\beta}} \triangleq\left[\beta_{1}, \beta_{2}, \cdots, \beta_{q}, 0\right.$, $\cdots, 0]^{T}, \overline{\boldsymbol{B}} \triangleq \operatorname{diag}\left(\beta_{1}, \beta_{2}, \cdots, \beta_{q}, 0, \cdots, 0\right)$, and $\tilde{\boldsymbol{R}}_{s} \triangleq \operatorname{diag}(0, \cdots, 0$, $\left.r_{s_{p+1}}, r_{s_{s+2}}, \cdots, r_{s_{n}}\right)$. We find that the ranks of the matrices in (16)-(19) still equal the number of incident signals.

\subsection{Direction Estimation without Eigendecomposition}

Because it is assumed that $M>2 p$ (i.e. $L-1>p$ ), we can divide the $(L-1) \times p$ matrix $\bar{A}(\theta)$ in (11) and (13)-(15) into two parts as follows

$$
\left.\bar{A}(\theta) \triangleq\left[\begin{array}{l}
A_{1}(\theta) \\
A_{2}(\theta)
\end{array}\right]\right\} p-2 p
$$

where $A_{2}(\theta)$ has the column $a_{2}\left(\theta_{k}\right) \triangleq\left[e^{j \omega_{p} p \tau\left(\theta_{k}\right)}\right.$, $\left.e^{j \omega_{0}(p+1) \tau\left(\theta_{k}\right)}, \cdots, e^{j \omega_{0}(L-2) r\left(\theta_{k}\right)}\right]^{T}$. Since $\bar{A}(\theta)$ and $A_{1}(\theta)$ are two submatrices of the Vandemonde matrix $A(\theta)$ with full rank in (3), the rows of $A_{2}(\theta)$ can be expressed as a linear combination of linearly independent rows of $A_{1}(\theta)$; equivalently there is a linear operator $P$ between $A_{1}(\theta)$ and $A_{2}(\theta)$ [6]

$$
P^{H} A_{4}(\theta)=A_{2}(\theta) \text {. }
$$

Hence it follows from (21) that

$$
Q^{H} \bar{A}(\theta)=O_{(M-2 p) \times p}
$$

where $Q \triangleq\left[P^{T},-I_{M-2 p}\right]^{T}$. As the $(L-1) \times(M-2 p)$ matrix $Q$ has a full rank of $M-2 p$, the columns of $Q$ in fact form the basis for the null space $\mathcal{N}\left(\bar{A}^{H}(\theta)\right)$ of $\bar{A}^{H}(\theta)$, and clearly the orthogonal projector onto this subspace is given by $\Pi_{Q} \triangleq Q\left(Q^{H} Q\right)^{-1} Q^{H}$ which implies that [15]

$$
\Pi_{Q} \bar{a}(\theta)=\mathbf{0}_{(L-1) \times 1}, \quad \text { for } \quad \theta=\theta_{k}
$$

where $\bar{a}(\theta) \triangleq\left[1, e^{j \omega_{n} \tau(\theta)}, \cdots, e^{j \omega_{0}(L-2) \tau(\theta)}\right]^{T}$, and $\mathbf{0}_{m \times 1}$ is an $m \times 1$ null vector. Evidently the directions can be estimated based on the orthogonal property (23) without any EVD/SVD.

Then the next problem is how to find the null space of $\overline{\boldsymbol{A}}^{H}(\theta)$ (i.e. $\boldsymbol{P}$ ) from the available array data. Based on the partition of $\bar{A}(\theta)$ in (20), the $(L-1) \times p$ correlation matrices $\Phi_{f}$ in (11), $\bar{\Phi}_{f}$ in (13), $\Phi_{b}$ in (14), and $\bar{\Phi}_{b}$ in (15) can be also divided as

$$
\begin{aligned}
& \left.\boldsymbol{\Phi}_{f}=\rho_{M} r_{s}\left[\begin{array}{l}
A_{1}(\theta) B A_{1}^{T}(\theta) \\
A_{2}(\theta) B A_{1}^{T}(\theta)
\end{array}\right] \triangleq\left[\begin{array}{l}
\Phi_{f 1} \\
\boldsymbol{\Phi}_{f 2}
\end{array}\right]\right\} M-2 p \\
& \left.\overline{\boldsymbol{\Phi}}_{f}=\rho_{1} r_{s}\left[\begin{array}{l}
\boldsymbol{A}_{1}(\theta) \boldsymbol{B} \boldsymbol{D} \boldsymbol{A}_{1}^{T}(\theta) \\
\boldsymbol{A}_{2}(\theta) \boldsymbol{B} \boldsymbol{D} \boldsymbol{A}_{1}^{T}(\theta)
\end{array}\right] \triangleq\left[\begin{array}{l}
\overline{\boldsymbol{\Phi}}_{f 1} \\
\overline{\boldsymbol{\Phi}}_{f 2}
\end{array}\right]\right\} M-2 p \\
& \left.\boldsymbol{\Phi}_{b}=\rho_{1}^{*} r_{s}\left[\begin{array}{l}
A_{1}(\theta) B^{*} D^{-(M-1)} A_{1}^{T}(\theta) \\
A_{2}(\theta) B^{*} D^{-(M-1)} A_{1}^{T}(\theta)
\end{array}\right] \triangleq\left[\begin{array}{l}
\boldsymbol{\Phi}_{b 1} \\
\boldsymbol{\Phi}_{b 2}
\end{array}\right]\right\} M-2 p \\
& \left.\overline{\boldsymbol{\Phi}}_{b}=\rho_{M}^{*} r_{s}\left[\begin{array}{l}
A_{1}(\theta) \boldsymbol{B}^{*} \boldsymbol{D}^{-(M-2)} A_{1}^{T}(\theta) \\
A_{2}(\theta) \boldsymbol{B}^{*} \boldsymbol{D}^{-(M-2)} A_{1}^{T}(\theta)
\end{array}\right] \triangleq\left[\begin{array}{l}
\overline{\boldsymbol{\Phi}}_{b 1} \\
\overline{\boldsymbol{\Phi}}_{b 2}
\end{array}\right]\right\} p-2 p
\end{aligned}
$$

From (21), we can obtain the following relation between the submatrices of $\Phi_{f}, \bar{\Phi}_{f}, \Phi_{b}$, and $\bar{\Phi}_{b}$

$$
P^{H} \Phi_{1}=\Phi_{2}
$$

where $\Phi_{1} \triangleq\left[\Phi_{f 1}, \bar{\Phi}_{f 1}, \Phi_{b 1}, \bar{\Phi}_{b 1}\right]$, and $\boldsymbol{\Phi}_{2} \triangleq\left[\Phi_{f 2}, \bar{\Phi}_{f 2}, \Phi_{b 2}, \bar{\Phi}_{b 2}\right]$. Thus the matrix $P$ can be found from $\Phi_{1}$ and $\Phi_{2}$ as

$$
\boldsymbol{P}=A_{1}^{-H}(\theta) A_{2}^{H}(\theta)=\left(\boldsymbol{\Phi}_{1} \boldsymbol{\Phi}_{1}^{H}\right)^{-1} \boldsymbol{\Phi}_{1} \boldsymbol{\Phi}_{2}^{H} .
$$

Proof: See [15] for details.

Therefore when the finite array data are available, the directions $\left\{\theta_{k}\right\}$ of coherent signals can be estimated in a manner similar to that of the MUSIC [3] by minimizing the following cost function

$$
f(\theta)=\bar{a}^{H}(\theta) \Pi_{\hat{Q}} \bar{a}(\theta)
$$

where $\Pi_{\hat{Q}}=\hat{Q}\left(\hat{Q}^{H} \hat{Q}\right)^{-1} \hat{Q}^{H}$, and $\hat{P}=\left(\hat{\Phi} \hat{\Phi}_{1}^{H}\right)^{-1} \hat{\Phi} \hat{\Phi}_{2}^{H}$.

\subsection{Implementation of SUMWE}

Based on the above analysis and by using the matrix inversion lemma, the implementation of the SUMWE for estimating the directions of coherent signals with the finite array data $\{y(n)\}_{n=1}^{N}$ can be summarized as follows:

a): Calculate the correlation vector $\hat{\varphi}$ between $y(n)$ and $y_{M}^{*}(n)$ and that $\hat{\bar{\varphi}}$ between $y(n)$ and $y_{i}^{*}(n)$ as

$$
\hat{\varphi}=\frac{1}{N} \sum_{n=1}^{N} y(n) y_{M}^{*}(n), \quad \hat{\varphi}=\frac{1}{N} \sum_{n=1}^{N} y(n) y_{1}^{*}(n)
$$

where $\hat{\varphi}=\left[\hat{r}_{M}, \hat{r}_{2 M}, \cdots, \hat{r}_{M M}\right]^{T}$, and $\hat{\bar{\varphi}}=\left[\hat{r}_{1}, \hat{r}_{21}, \cdots, \hat{r}_{M}\right]^{T}$

b): Form the estimated matrices $\hat{\Phi}_{f}, \hat{\Phi}_{f}, \hat{\Phi}_{b}$, and $\hat{\Phi}_{b}$ from $\hat{\varphi}$ and $\hat{\varphi}$ by using (11) and (13)-(15).

c): Estimate the linear operator $\boldsymbol{P}$ as

$$
\hat{P}=\left(\hat{\Phi} \hat{\Phi}_{1}^{H}\right)^{-1} \hat{\Phi}_{2} \hat{\Phi}_{2}^{H}
$$

and calculate the orthogonal projector $\Pi_{\hat{Q}}$ as

$$
\Pi_{\hat{Q}}=\hat{Q}\left(I_{M-2 p}-\hat{\boldsymbol{P}}^{H}\left(\hat{\boldsymbol{P}} \hat{\boldsymbol{P}}^{H}+I_{p}\right)^{-1} \hat{\boldsymbol{P}}\right) \hat{\boldsymbol{Q}}^{H} .
$$

d): Estimate the directions $\left\{\theta_{k}\right\}$ by searching the $p$ highest peaks of the spatial spectrum $P(\theta)$ or by finding the phases of the $p$ zeros of the polynomial $p(z)$ closest to the unit circle in the $z$-plane, where $P(\theta) \triangleq 1 / \bar{a}^{H}(\theta) \Pi_{\hat{Q}} \bar{a}(\theta), \quad p(z) \triangleq z^{L-2} p^{H}(z) \Pi_{\hat{Q}} p(z), \quad p(z)=$ $\left[1, z, \cdots, z^{L-2}\right]^{T}$, and $z \triangleq e^{j \omega_{0} \tau(\theta)}$.

Remark B: By defining the array covariance matrix $\boldsymbol{R}$ as $\boldsymbol{R} \triangleq E\left\{\boldsymbol{y}(n) \boldsymbol{y}^{H}(n)\right\}=A(\theta) \boldsymbol{R}_{s} A^{H}(\theta)+\sigma^{2} \boldsymbol{I}_{M}$, where $\boldsymbol{R}_{s}$ $\triangleq E\left\{s(n) s^{H}(n)\right\}$, we can find that the matrices $\boldsymbol{\Phi}_{f}, \overline{\boldsymbol{\Phi}}_{f}, \boldsymbol{\Phi}_{b}$, and $\bar{\Phi}_{b}$ are formed from the correlations in the $M$ th and 1st columns and those in the 1st and $M$ th rows of $R$ except for the correlations $r_{11}$ and $r_{M M}$, which contain the variance $\sigma^{2}$ of additive noise. Hence the actual number of needed correlations $\left\{r_{i k}\right\}$ is $2 M-3$, because the matrix $R$ is Hermitian.

Remark C: The SUMWE can accommodate a more general noise model of the spatially correlated noise if we choose the signal vectors $y_{f l}(n)$ and $y_{b l}(n)$ used to form the matrices $\bar{\Phi}_{f}, \bar{\Phi}_{f}, \Phi_{b}$, and $\bar{\Phi}_{b}$ appropriately [15]. 口

Remark D: The estimated number of MATLAB flops required by the SUMWE algorithm is nearly $16 \mathrm{NM}+$ $16 M(M-p)^{2}$ [15], when $N \gg M \gg p$ which occurs often in applications of DOA estimation, where the computations needed in the remaining steps are negligible.

Remark E: As the SUMWE estimator is a complicated nonlinear function of the received array data, its statistical 
behaviour appears to be difficult to analyze. For a large number of snapshots, the asymptotic properties of the SUMWE can be obtained (see [15] and [20] for details).

\section{Adaptive Algorithm for Direction Estimation}

\subsection{Adaptive Estimation of Null Space}

Now we consider the real-time implementation of the SUMWE for tracking the slowly time-varying directions of moving signals. First, the estimation of linear operator $P(n)$ at the time $n$ can be reduced to the minimization of the time-varying cost function $J(n)$ given by

$$
J(n) \triangleq\|E(n)\|^{2}=\operatorname{tr}\left\{E^{H}(n) E(n)\right\}
$$

where $E(n)$ is the estimation error given by $E(n) \triangleq$ $\Phi_{2}^{H}(n)-\Phi_{7}^{H}(n) P(n-1),\|\cdot\|^{2}$ and $\operatorname{tr}\{\cdot\}$ denote the square of the Frobenius norm and the trace operation.

By defining the derivative of $J \triangleq f(z)$ with respect to a complex variable vector $z \triangleq\left[z_{1}, z_{2}, \cdots, z_{m}\right]^{T}$ and the gradient vector of $J$ as [19]

$$
\begin{aligned}
& \frac{\partial J}{\partial z^{*}} \triangleq \frac{1}{2}\left[\frac{\partial J}{\partial x_{1}}+j \frac{\partial J}{\partial y_{1}}, \cdots, \frac{\partial J}{\partial x_{m}}+j \frac{\partial J}{\partial y_{m}}\right]^{T} \\
& \nabla J \triangleq\left[\frac{\partial J}{\partial x_{1}}+j \frac{\partial J}{\partial y_{1}}, \cdots, \frac{\partial J}{\partial x_{m}}+j \frac{\partial J}{\partial y_{m}}\right]^{T}
\end{aligned}
$$

where $z_{k} \triangleq x_{k}+j y_{k}$, and using the facts for matrices $A$ and $X$ with compatible dimensions that

$$
\frac{\partial\left(\operatorname{tr}\left\{X^{H} A\right\}\right)}{\partial X^{*}}=A, \frac{\partial(\operatorname{tr}\{A X\})}{\partial X^{*}}=O, \frac{\partial\left(\operatorname{tr}\left\{X^{H} A X\right\}\right)}{\partial X^{*}}=A X
$$

we can obtain

$$
\frac{\partial J(n)}{\partial P^{\bullet}(n-1)}=-\Phi_{1}(n)\left(\Phi_{2}^{H}(n)-\Phi_{1}^{H}(n) P(n-1)\right) .
$$

Hence the instantaneous gradient matrix of $J(n)$ in (34) with respect to $P(n-1)$ is given by

$$
\nabla J(n)=-2 \frac{\partial J(n)}{\partial P^{*}(n-1)}=-2 \Phi(n) E(n) .
$$

Thus we easily have the least-mean-square (LMS) algorithm for updating the linear operator $P(n)$ [19]

$$
\boldsymbol{P}(n)=\boldsymbol{P}(n-1)-0.5 \mu \nabla J(n)=\boldsymbol{P}(n-1)+\mu \Phi(n) \boldsymbol{E}(n)
$$

where $\mu$ is the step-size, and it should be chosen to satisfy $0<\mu<2 / \lambda_{\max }$, where $\lambda_{\max }$ is the largest eigenvalue of $E\left\{\Phi_{\{}(n) \Phi_{\rightarrow}^{H}(n)\right\}$. Then from (33), we can obtain the instantaneous orthogonal projector onto the null space $\mathcal{N}\left(\bar{A}^{H}(\theta)\right)$

$$
\begin{aligned}
\Pi(n)= & Q(n)\left(I_{M-2 p}-P^{H}(n)\right. \\
& \left.\cdot\left(P(n) P^{H}(n)+I_{p}\right)^{-1} P(n)\right) Q^{H}(n)
\end{aligned}
$$

where $Q(n)=\left[\mathbb{P}^{T}(n),-\boldsymbol{I}_{M-2 p}\right]^{T}$.

Although the matrix inversion lemma is used to reduce the computational complexity in the calculation of orthogonal projector in (40), where the inversion of a $p \times p$ matrix $P(n) P^{H}(n)+I_{p}$ is involved instead of a $(M-2 p) \times(M-2 p)$ matrix $Q(n) Q^{H}(n)$, this inversion is unsuitable for the real-time application. Due to the computational expediency of the Householder transformation, which is simply a reflection operation to annihilate all the elements but a column except for the first one, we perform the $\mathrm{QR}$ decomposition of $P(n) P^{H}(n)+I_{p}$ with the Householder transformation

$$
P(n) P^{H}(n)+I_{p} \triangleq \bar{P}=\bar{Q} \bar{R}
$$

where $\bar{Q}$ is a $p \times p$ unitary matrix, and $\bar{R}$ is a $p \times p$ upper-triangular matrix. Then the instantaneous orthogonal projector $\Pi(n)$ can be obtained

$$
\Pi(n)=Q(n)\left(I_{M-2 p}-P^{H}(n) R^{-1} \bar{Q}^{H} P(n)\right) Q^{H}(n)
$$

Note that the inversion $\bar{R}^{-1}$ is easily got by a simple back-substitution, because the matrix $\bar{R}$ is an uppertriangular matrix

\subsection{Approximate Newton's Iteration Method for Direction Estimation}

As the estimate $\hat{\theta}_{k}$ is obtained by minimizing $f(\theta)$ in (30) and it is a consistent estimate [15], [20], the derivative of $f(\theta)$ can be approximated using two terms in its Taylor series expansion about the true value $\theta_{k}$ as

$$
0=f^{\prime}\left(\hat{\theta}_{k}\right) \approx f^{\prime}\left(\theta_{k}\right)+f^{\prime \prime}\left(\theta_{k}\right)\left(\hat{\theta}_{k}-\theta_{k}\right)
$$

where the second- and higher order terms in (43) can be neglected, and the first- and second-order derivatives of $f(\theta)$ with respect to $\theta$ are given by

$$
\begin{aligned}
f^{\prime}(\theta) & =d f(\theta) / d \theta=2 \operatorname{Re}\left\{\bar{d}^{H}(\theta) \Pi_{\hat{Q}} \bar{a}(\theta)\right\} \\
f^{\prime \prime}(\theta) & =d f^{\prime}(\theta) / d \theta \\
& =2 \operatorname{Re}\left\{\tilde{d}^{H}(\theta) \Pi_{\hat{Q}} \bar{a}(\theta)+\bar{d}^{H}(\theta) \Pi_{\hat{Q}} \bar{d}(\theta)\right\}
\end{aligned}
$$

where $\bar{d}(\theta)=d \bar{a}(\theta) / d \theta$, and $\tilde{d}(\theta)=d \bar{d}(\theta) / d \theta$.

From (43), the first-order expression for the estimation error of direction $\theta_{k}$ can be obtained as

$$
\hat{\theta}_{k}-\theta_{k}=-\frac{f^{\prime}\left(\theta_{k}\right)}{f^{\prime \prime}\left(\theta_{k}\right)} \approx-\frac{\operatorname{Re}\left\{\bar{d}^{H}\left(\theta_{k}\right) \Pi_{\hat{Q}} \bar{a}\left(\theta_{k}\right)\right\}}{\bar{d}^{H}\left(\theta_{k}\right) \Pi_{Q} \bar{d}\left(\theta_{k}\right)}
$$

where the estimated orthogonal projector $\Pi_{\hat{Q}}$ in the denominator of (46) can be replaced with the true one $\Pi_{O}$ without affecting the asymptotic property of estimate $\hat{\theta}_{k}$ [15]. Thus we have the approximate Newton's iteration method for direction estimation as [19]

$$
\hat{\theta}_{k}(n)=\hat{\theta}_{k}(n-1)-\left.\frac{\operatorname{Re}\left\{\bar{a}^{H}(\theta) \Pi(n) d(\theta)\right\}}{d^{H}(\theta) \Pi(n) d(\theta)}\right|_{\theta=\hat{\theta}_{k}(n-1)} .
$$

\subsection{Adaptive Algorithm for DOA Estimation and Tracking}

Although the LMS algorithm with a nonvanishing step-size described in Section 4.1 has its computational simplicity and good convergence property in the stationary environment, the determination of an appropriate step-size is rather difficult for tracking the time-varying directions of incident signals. Based on the adaptive estimation of null space and iteration of direction described above, we can summarize the adaptive algorithm for estimating the timevarying directions of incidents signals as follows, where a modified LMS is used in the estimation of null space for improving the tracking perfromance.

1): Calculate the instantaneous correlation vector $\varphi(n)$ between $y(n)$ and $y_{M}^{*}(n)$ and that $\bar{\varphi}(n)$ between $y(n)$ and $y_{1}^{*}(n)$ as

$$
\varphi(n)=y(n) y_{M}^{*}(n), \quad \bar{\varphi}(n)=y(n) y_{1}^{*}(n)
$$

where $\varphi(n)=\left[\hat{r}_{M}(n), \hat{P}_{2 M}(n), \cdots, \hat{P}_{M M}(n)\right]^{T}$, and $\bar{\varphi}(n)=$ $\left[P_{11}(n), P_{21}(n), \cdots, P_{M 1}(n)\right]^{T}$. 
2): Form the instantaneous estimates of Hankel correlation matrices $\Phi_{f}(n), \Phi_{f}(n), \Phi_{b}(n)$, and $\bar{\Phi}_{b}(n)$ from $\varphi(n)$ and $\bar{\varphi}(n)$ as

$$
\begin{array}{ll}
\Phi_{f}(n)=\operatorname{Hank}\left\{h_{c}, h_{r}\right\}, & \bar{\Phi}_{f}(n)=\operatorname{Hank}\left\{\bar{h}_{c}, \bar{h}_{r}\right\} \\
\Phi_{b}(n)=J_{L-1} \bar{\Phi}_{f}^{*}(n) J_{p}, & \bar{\Phi}_{b}(n)=J_{L-1} \Phi_{f}^{*}(n) J_{p}
\end{array}
$$

where $h_{c}=\left[\hat{P}_{M}(n), \hat{P}_{2 M}(n), \cdots, \hat{r}_{M-p, M}(n)\right]^{T}, \quad h_{r}=\left[P_{M-p, M}(n)\right.$, $\left.\hat{P}_{M-p+1, M}(n), \cdots, P_{M-1, M}(n)\right]^{T}, \quad \bar{h}_{c}=\left[P_{21}(n), P_{31}(n), \cdots, \hat{P}_{L 1}(n)\right]^{T}$, $\bar{h}_{r}=\left[P_{L 1}(n), \hat{P}_{L+1,1}(n), \cdots, P_{M 1}(n)\right]^{T}$, and Hank $\{\cdot\}$ denotes the Hankel operation.

3): Update the linear operator $P(n)$ as

$$
P(n)=P(n-1)+\bar{\mu} \frac{\Phi_{1}(n)\left(\Phi_{2}^{H}(n)-\Phi_{1}^{H}(n) P(n-1)\right)}{\operatorname{tr}\left\{\Phi_{1}(n) \Phi_{1}^{H}(n)\right\}}
$$

where $\bar{\mu}$ is the step-size, and $0<\bar{\mu}<2$.

4): Calculate an auxiliary matrix as $\overline{\boldsymbol{P}}=\boldsymbol{P}(n) \boldsymbol{P}^{H}(n)+\boldsymbol{I}_{p}$, and perform the QR factorization of $\bar{P}$ based on Householder transformation as (41).

5): Form the projector as $Q(n)=\left[P^{T}(n),-I_{M-2 p}\right]^{T}$, and calculate the orthogonal projector $\Pi(n)$ as (42).

6): Update the estimates of directions $\left\{\hat{\theta}_{k}(n)\right\}$ by using the approximate Newton's iteration method as (47).

In addition, the (modified) LMS algorithm is initialized by $P(0)=O_{p \times(M-2 p)}$, and the first the first $K_{0}=2 M$ snapshots of the received data are accumulated for an off-line SUMWE to provide the initial values of directions $\left\{\hat{\theta}_{k}(n)\right\}$ for the Newton's iteration method.

\section{Numerical Examples}

The ULA with $M$ sensors is separated by a half-wavelength, and two signals with equal power come from angles $\theta_{1}$ and $\theta_{2}$. The SNR is defined as the ratio of the power of the source signals to that of the additive noise at each sensor. The results shown below are all based on 1000 independent trials.

\section{Example 1: Performance of SUMWE versus SNR}

The incident directions of two coherent signals are $\theta_{1}=5^{\circ}$ and $\theta_{2}=12^{\circ}$, and their SNR is varied from -10 to $25 \mathrm{~dB}$. The number of sensors is $M=10$, and the number of snapshots is $N=128$. Additionally the subarray size is set as $m=7$ for the SS-based algorithms [10], [14].

The empirical root MSEs (RMSEs) of the estimates $\hat{\theta}_{1}$ and $\hat{\theta}_{2}$ are shown in Fig. 1, where the theoretical RMSEs of the SUMWE (see [15] for details) and the stochastic Cramér-Rao lower bounds (CRBs) [17] are also plotted. Because the maximum possible number of subarrays and working array aperture are exploited and the effect of additive noise is eliminated by appropriately choosing the used subarrays, the proposed SUMWE method generally outperforms the SS-based root-MUSIC with EVD [3], [10], [16] and the methods without EVD such as the BEWE (variant for coherent signals) [5] and FBSS-based SWEDE (variant G) [7], [14], and it is superior to the FBSS-based OPM without EVD [6], [14] at low SNRs. Although the WSF-E [8] performs better at high SNR, its performance degrades severely and its RMSEs are larger than those of the SUMWE at low to moderate SNRs. From Fig. 1, we can see that the empirical RMSEs of the SUMWE are very close to the theoretical ones (except at low SNR) and the difference between the theoretical RMSEs and the CRBs is small. And the proposed SUMWE is computationally efficient, and the simulation shows that the ratio of the number of MATLAB flops required by the SS-based MUSIC and the WSF-E to that required by the SUMWE is about 7.147 and 18.677 .
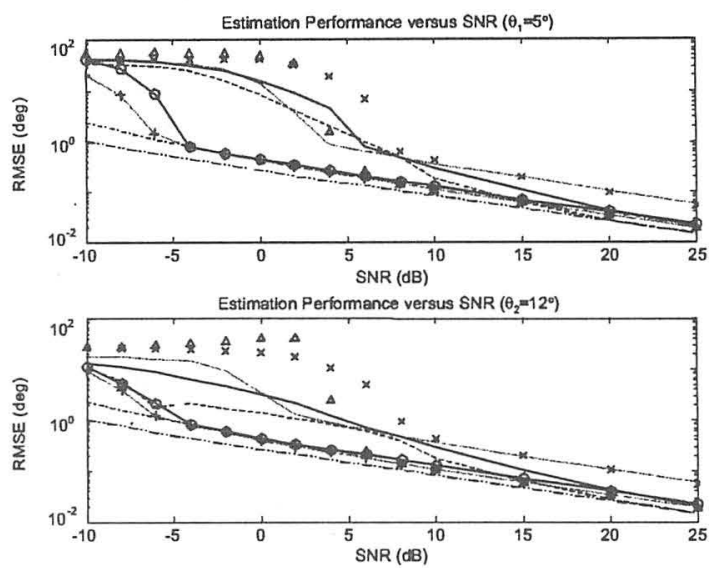

Fig. 1 RMSEs of the estimates versus the SNR (dotted line: SS-based root-MUSIC; dotted line with "+": FBSS-based root-MUSIC; " $\triangle$ ": FBSS-based OPM; " $"$ ": BEWE; solid line: FBSS-based SWEDE; dashed line: WSF-E; solid line with "o": SUMWE; dash-dot line: theoretical RMSE of SUMWE; and dash-dots line: CRB).

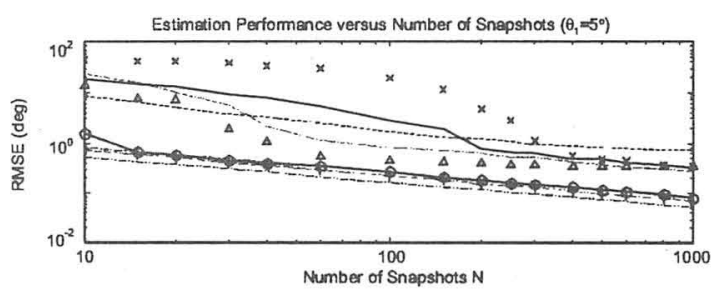

Estimation Performence versus Number of Snepshots $\left(\theta_{2}=12^{\circ}\right)$

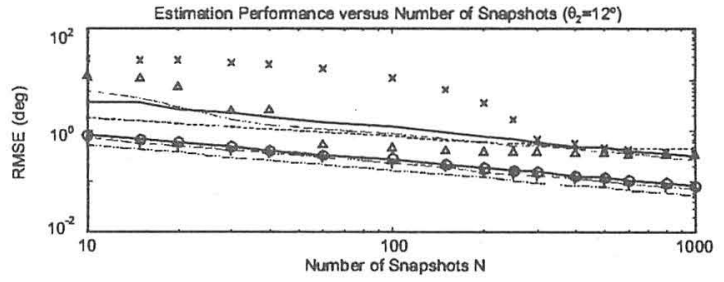

Fig. 2 RMSEs of the estimates versus the number of snapshots (dotted line: SS-based root-MUSIC; dotted line with "+": FBSS-based root-MUSIC; " $\triangle$ ": FBSS-based OPM; " $x$ ": BEWE; solid line: FBSS-based SWEDE; dashed line: WSF-E; solid line with "o": SUMWE; dash-dot line: theoretical RMSE of SUMWE; and dash-dots line: CRB).

\section{Example 2: Performance of SUMWE versus Number of Snapshots}

The simulation conditions are similar to those in Example 1, except that the SNR is set at $5 \mathrm{~dB}$, and the number of snapshots is varied from $N=10$ to $N=1000$.

The empirical RMSEs of the estimated directions $\hat{\theta}_{1}$ and $\hat{\theta}_{2}$ are plotted in Fig. 2, and they are compared with the theoretical RMSEs of the SUMWE and the CRBs. We can see that the SUMWE is superior to the SS-based root-MUSIC, BEWE, FBSS-based OPM, FBSS-based SWEDE, and WSF-E even for a small number of snapshots. Furthermore, we can find that the empirical RMSEs agree very well with the theoretical RMSEs derived in Section 4 (their difference is almost indistinguishable) and the 
empirical and theoretical RMSEs of the SUMWE decrease monotonically with the number of snapshots.

\section{Example 3: Adaptive Tracking of Time-Varying DOAs}

Here two coherent signals with equal power come from $\theta_{1}(n)=30^{\circ}+0.01^{\circ}(n-1)$ and $\theta_{2}(n)=10^{\circ}+5^{\circ} \sin \left(2 \pi\left(4 \times 10^{-4} n\right.\right.$ $\left.\left.+2.25 \times 10^{-6} n^{2}\right)\right)$, where $n=1,2, \cdots, 1000$, and the SNR is $20 \mathrm{~dB}$. The number of sensors is $M=16$, the step-size of modified LMS algorithm is set as $\bar{\mu}=1$, and the first $K_{0}=2 M$ snapshots of the received data is used to determine the initial values of directions via the grid method of SUMWE. The proposed on-line algorithm is carried out, and the averaged estimates and estimation errors are shown in Figs. 3 and 4. Even though the SS and eigendecomposition processes are not used, the proposed algorithm can promptly track the variation in the desired directions of coherent signals with less estimation errors.

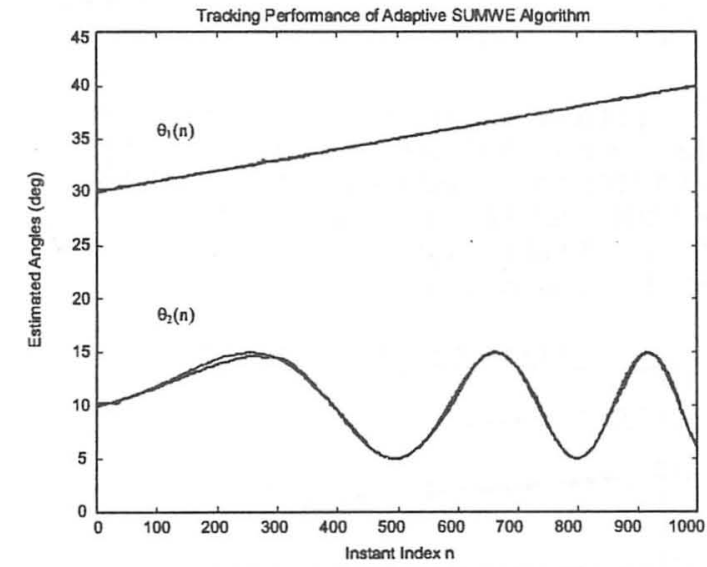

Fig. 3 Averaged estimates of time-varying directions coherent signals (dotted line: actual value, and solid line: proposed algorithm)
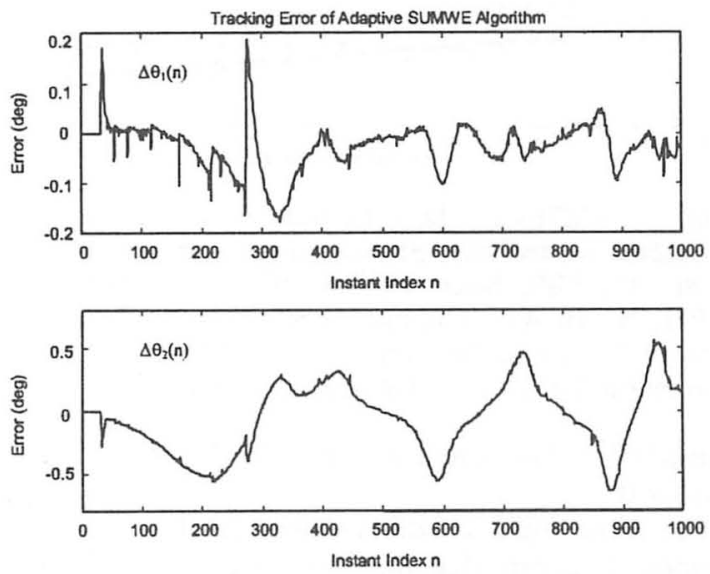

Fig. 4 Averaged estimation errors of estimated time-varying directions.

\section{Conclusion}

A new computationally efficient subspace-based method called SUMWE for direction estimation of narrow-band signals impinging on a ULA by exploiting the array geometry and its shift invariance property, and its adaptive implementation was presented for tracking the directions of moving signals. The effectiveness of the proposed
SUMWE and its adaptive implementation were verified through numerical examples. It was shown that the proposed adaptive algorithm has the advantages of the computational simplicity and good tracking adaptation in a slowly time-varying environment.

References:

[1] H.L. Van Trees: Optimum Array Processing, Part IV of Detection, Estimation, and Modulation Theory. New York: NY, John Wiley \& Sons (2002)

[2] H. Krim \& M. Viberg: Two decades of array signal processing research: The parametric approach, IEEE Signal Processing Mag., 13 4, $67 / 94$ (1996)

[3] R.O. Schmidt: Multiple emitter location and signal parameter estimation, IEEE Trans. Antennas Propagat., 34 3, 276/280 (1986)

[4] P. Comon \& G.H. Golub: Tracking a few extreme singular values and vectors in signal processing, Proc. IEEE, 78 8, 1327/1343 (1990)

[5] C.-C. Yeh: Simple computation of projection matrix for bearing estimations, IEE Proc., Pt. F, 134 2, 146/150 (1987)

[6] S. Marcos, A. Marsal \& M. Benidir: The propagator method for source bearing estimation, Signal Processing, 42 2, 121/138 (1995)

[7] A. Eriksson, P. Stoica \& T. Söderström: On-line subspace algorithms for tracking moving sources, IEEE Trans. Signal Processing, 42 9, 2319/2330 (1994)

[8] M. Kristensson, M. Jansson \& B. Ottersten: Modified IQML and weighted subspace fitting without eigendecomposition, Signal Processing, 79 1, 29/44 (1999)

[9] P. Stoica \& T. Söderström: Statistical analysis of a subspace method for bearing estimation without eigendecomposition, IEE Proc., Pt. F, 139 4, 301/305 (1992)

[10] T.-J. Shan, M. Wax \& T. Kailath: On spatial smoothing for direction-of-arrival estimation of coherent signals, IEEE Trans. Acoust., Speech, Signal Processing, 33 4, 806/811 (1985)

[11] J. Xin \& A. Sano: MSE-based regularization approach to direction estimation of coherent narrowband signals using linear prediction, IEEE Trans. Signal Processing, $4911,2481 / 2497$ (2001)

[12] J. Xin \& A. Sano: Linear prediction approach to direction estimation of cyclostationary signals in multipath environment, IEEE Trans. Signal Processing, 49 4, $710 / 720(2001)$

[13] J. Xin \& A. Sano: Direction estimation of coherent signals using spatial signature, IEEE Signal Processing Letters, 9 12, 414/417 (2002)

[14] S.U. Pillai \& B.H. Kwon: Forward/backward spatial smoothing techniques for coherent signals identification, IEEE Trans. Acoust., Speech, Signal Processing, 37 1, $8 / 15(1989)$

[15] J. Xin \& A. Sano: Computationally efficient subspacebased method for direction-of-arrival estimation without eigendecomposition, IEEE Trans. Signal Processing, 52 4 (2004) (to appear)

[16] A. J. Barabell: Improving the resolution performance of eigenstructure-based direction-finding algorithms, Proc. IEEE Int. Conf. Acoust., Speech, Signal Processing, 1, $336 / 339(1983)$

[17] P. Stoica \& A. Nehorai: Performance study of conditional and unconditional direction-of-arrival estimation, IEEE Trans. Acoust., Speech, Signal Processing, 38 10, 1783/1795 (1990)

[18] J.C. Liberti Jr \& T.S. Rappaport: Smart Antennas for Wireless Communications: IS-95 and Third Generation CDMA Applications. Upper Saddle River, NJ: Prentice-Hall (1999)

[19] S. Haykin: Adaptive Filter Theory, 4th edition. Upper Saddle River, NJ: Prentice-Hall (2002)

[20] J. Xin \& A. Sano: Statistical analysis of subspace-based method for direction estimation without eigendecomposition, Proc. IFAC 13th Symp. System Identification, 1078/1083 (2003) 